\title{
Data adaptive control parameter estimation for scaling laws for magnetic fusion devices
}

\author{
R. Preuss, H. Dreier, A. Dinklage, and V. Dose \\ Max-Planck-Institut für Plasmaphysik, EURATOM-Association \\ Wendelsteinstr. 1, Greifswald, Germany, EU
}

\begin{abstract}
Data adaptive planing determines the expected utility of a single new measurement using existing data and a data descriptive model. The method can be used for experimental planning. It is applied to scaling laws for magnetic fusion devices. Explicitly, the scaling of the stellarator W7-AS is examined for a subset of $t=1 / 3$ data. In control parameter space regions of high utility are identified and serve for fixing discharge and machine parameters for upcoming discharges. It will be shown that a skillfull analysis of experimental uncertainties is of utmost importance for significant results.
\end{abstract}

PACS 52.55.-s Magnetic confinement and equilibrium

PACS 52.25.Fi Transport Properties

PACS 02.50.-r Probability theory, stochastic processes, and statistics

\section{Motivation: Planning of fusion experiments}

A prominent task in experimental sciences is the choice of settings for future measurements. This issue becomes crucial if experimental access is expensive or limited.

Examples particularly motivating this study are experiments in nuclear fusion devices. In magnetic confinement fusion experiments, a large number of different data from a couple of diagnostics are taken to derive data for physics inferences. For the ITER device, the largest thermonuclear magnetic confinement device planned [ITER team(1999)], the burning fusion plasma 
will also result in an expected limitation of experimental time due to its harsh experimental conditions. But even for existing devices, the limitation of experimental time motivates procedures which allow the validation of physics models at highest possible efficiency.

¿From an abstract point of view, experimental planning may be regarded as the optimal choice of control parameters for the assessment of a physical model, i.e., the response to these control parameters. Hence, the physical model is part of the planning strategy.

For fusion devices, the parameter space of settings is not only limited but even structured. Certain combinations of control parameters (e.g heating power and density) require more effort than other ones. Consequently, the question arises which data contribute to the validation of a hypothesis most and which data are redundant. The answer to this question may prevent the experimentalist to repeat measurements or to choose parameters which result in a setting with small informational content.

In the example discussed here, the physical model is a scaling law for the energy confinement time $\tau_{e}$. Scaling laws are used for estimates of device performance and have proven to be extremely useful for planning and design of new devices. $\tau_{e}$ is a measure of thermal insulation of a particular magnetic configuration and its plasmas. The confinement time is derived from the stored plasma energy and the deposited heating power $\tau_{e}=W / P$. Scaling laws for the energy confinement in magnetic fusion devices are formulated as functional dependencies of the energy content $W$ on control parameters. The plasma confinement can be controlled by gas flows, heating power, the magnetic configuration and geometry of the device. While change of geometry meets with difficulties during experiments, the other control parameters allow wide variations in parameter space.

With this background, two issues are addressed in this paper for the first time: (1) the assessment of relevance of data, (2) planning of experimental campaigns in nuclear fusion by means of Bayesian Experimental Planning [Lindley(1956)]. The proposed method assumes an existing set of control parameters for confinement measurements and employs information measures to quantify the impact of a new measurement. Since the method is formulated very generally, the approach is relevant to other disciplines of experimental sciences apart from plasma physics.

Specifically, the method uses an expected utility function, the kernel of which is chosen to be the information gain due to the measurement. As data adaptive exploration (DAE) we define the evaluation of individual expected 
utilities for existing data, data adaptive planning (DAP) is the maximization of the expected utility with respect to the control parameters for a future experiment. The use of Bayesian experimental design for the planning of future experiments was put forward by $[\operatorname{Loredo}(2003)]$ to estimate the optimum observation time for orbital parameters of extra-solar planets. An example in fusion is the choice of optimum lines of sight for an interferometer [Dreier et al.(2006)].

In this paper, the method of data adaptive planning is applied to a multivariate linear case. A prominent result of this study is the impact of a proper uncertainty treatment. Considering improper uncertainties, or even the neglect of uncertainties, leads to wrong results.

\section{Scaling Laws for Fusion Devices}

Scaling laws for fusion devices are a predestined field for application of DAP and DAE: First, data bases containing several hundred data sets exist (e.g. the International Stellarator Confinement Data Base (ISCDB) [Yamada et al.(2005)]) and can be analysed with respect to individual data. Second, it is possible to plan experiments at running machines. And, finally, the scalings are also used to design future devices.

In the special case of a logarithmic scaling law, the DAP/DAE approach for a multi dimensional linear problem can be used.

\subsection{Background}

Scaling laws connect plasma and machine parameters with quantities relevant for plasma and energy confinement. They are widely used for inter-machine comparison of different discharges and prediction of the confinement performance of future experiments. The first point is motivated by the limitations in the parameter range for a single experiment (e.g. restrictions in geometrical variations). This can be overcome by a comparison of different machines. The second application arises from the fact that transport mechanisms in fusion plasmas are not fully understood in the sense that the functional dependence of the energy content $W$ on the experimental parameters density $n$, magnetic field $B$, effective minor radius $a$, major radius $R$ and total heating power $P$ and the quantity describing the plasma confinement cannot be calculated from first principles. 
For toroidal magnetic fusion devices, semi-empirical scalings are used instead. For a stellarator with given major radius $R$ and fixed rotational transform this relation reads:

$$
W=\exp \left\{\alpha_{c}\right\} a^{\alpha_{a}} P^{\alpha_{P}} n^{\alpha_{n}} B^{\alpha_{B}} .
$$

The $\alpha_{k}$ are the scaling exponents describing the dependencies of $W$ on the scaling parameters. In most cases these scaling parameters have been found by regression procedures from available data sets. Commonly, the logarithm is applied to linearize Eq. (1)

$$
\ln W=\sum_{k} \alpha_{k} x_{k}
$$

with $\boldsymbol{x}=\{1, \ln a, \ln P, \ln n, \ln B\}^{T}$. It has been shown previously that this transformation allows for unbiased estimates for the coefficients of $\boldsymbol{x}$ [Dose et al.(1996)], provided that uncertainties in $a, P, n$ and $B$ are transformed accordingly.

\section{Bayesian Probability Theory}

The Bayesian approach [Jaynes(2005)] is a powerful and self-consistent inference framework to analyse data and to design experiments. It rests on the product and sum rule of probability theory for the treatment of conditional probability distribution functions (PDF). An immediate consequence of these rules is Bayes theorem

$$
p(\boldsymbol{\alpha} \mid \boldsymbol{d}, I)=\frac{p(\boldsymbol{\alpha} \mid I) p(\boldsymbol{d} \mid \boldsymbol{\alpha}, I)}{p(\boldsymbol{d} \mid I)} .
$$

The prior knowledge about the model parameters $\boldsymbol{\alpha}$ encoded in the probability distribution $p(\boldsymbol{\alpha} \mid I)$ is modified by the likelihood of $\boldsymbol{\alpha}$ due to the newly accumulated data $\boldsymbol{d}, p(\boldsymbol{d} \mid \boldsymbol{\alpha}, I)$ to obtain the posterior distribution of $\boldsymbol{\alpha}, p(\boldsymbol{\alpha} \mid \boldsymbol{d}, I)$. The condition $I$ summarises all other information related to the problem which need not be made explicit for the intended calculation. The denominator in (3), $p(\boldsymbol{d} \mid I)$, ensures normalisation of $p(\boldsymbol{\alpha} \mid \boldsymbol{d}, I)$.

$$
p(\boldsymbol{d} \mid I)=\int \mathrm{d} \boldsymbol{\alpha} p(\boldsymbol{\alpha} \mid I) p(\boldsymbol{d} \mid \boldsymbol{\alpha}, I)=\int \mathrm{d} \boldsymbol{\alpha} p(\alpha, \boldsymbol{d} \mid I) .
$$

The second equality in (4) results from the above mentioned product rule. Eq. (4) is also known as the Bayesian marginalisation rule and allows to 
remove variables from a calculation which may be necessary for the initial formulation of the problem but are of no interest per se. Both, Eqn. (3) and (4), are used repeatedly in the following.

The goal of the present work is the identification of optimal operation parameters for the information gain on energy confinement of a toroidal magnetic confinement fusion device. Let $W$ denote the energy stored in the plasma, $\boldsymbol{x}$ the vector of operation parameters and $\boldsymbol{r}$ the error associated with the measurement of $W$. For a data set of $N$ measurements $\boldsymbol{d}=\boldsymbol{W}$ and $\boldsymbol{r}$ become vectors and the operation parameters are represented by the matrix $\mathbf{X}=\left\{\boldsymbol{x}_{1}, \boldsymbol{x}_{2}, \ldots, \boldsymbol{x}_{N}\right\}^{T}$. The model equation for explaining the data is then

$$
d-\mathrm{X} \cdot \boldsymbol{\alpha}=\boldsymbol{r} .
$$

In planning an experiment one is interested in the utility of the next data point $D$, described by the same linear model as above

$$
D-\boldsymbol{\xi}^{T} \cdot \boldsymbol{\alpha}=\rho,
$$

where $\rho$ is the measurement error of the datum $D$. Our interest is in the optimal choice of the experimental settings $\boldsymbol{\xi}=\{1, \ln \hat{a}, \ln \hat{P}, \ln \hat{n}, \ln \hat{B}\}^{T}$ for this new datum $D$. Following [Lindley(1956)] and [Loredo(2003)] we define the information gain, hence forth called utility, of $(D, \boldsymbol{\xi})$ as the Kullback-Leibler distance [Kapur and Kesavan(1992)] between the posterior distribution of $\boldsymbol{\alpha}$ with and without the new datum $D$.

$$
U(D, \boldsymbol{\xi})=\int \mathrm{d} \boldsymbol{\alpha} p(\boldsymbol{\alpha} \mid D, \boldsymbol{d}, \boldsymbol{\xi}, \mathbf{X}, I) \ln \frac{p(\boldsymbol{\alpha} \mid D, \boldsymbol{d}, \boldsymbol{\xi}, \mathbf{X}, I)}{p(\boldsymbol{\alpha} \mid \boldsymbol{d}, \mathbf{X}, I)} .
$$

The Expected Utility (EU) is the expectation value of the utility function over the data space for $D$ :

$$
E U(\boldsymbol{\xi})=\int \mathrm{d} D p(D \mid \boldsymbol{d}, \boldsymbol{\xi}, \mathbf{X}, I) U(D, \boldsymbol{\xi}) .
$$

By virtue of the marginalization rule the first term in the above integral is

$$
p(D \mid \boldsymbol{d}, \boldsymbol{\xi}, \mathbf{X}, I)=\int \mathrm{d} \boldsymbol{\alpha} p(\boldsymbol{\alpha} \mid \boldsymbol{d}, \mathbf{X}, I) p(D \mid \boldsymbol{\alpha}, \boldsymbol{\xi}, I) .
$$

In order to evaluate (9) we need to specify the two distributions under the integrand. This is simplest for the second term. If we know $\boldsymbol{\alpha}$ and $\boldsymbol{\xi}$ then $D$ follows from the data description model and is given by

$$
p(D \mid \boldsymbol{\alpha}, \boldsymbol{\xi}, I)=\delta\left(D-\boldsymbol{\xi}^{T} \boldsymbol{\alpha}\right) .
$$


The first term in (9) on the other hand is the posterior distribution of $\boldsymbol{\alpha}$ given only the data set $\boldsymbol{d}$. Assuming a flat prior for $\boldsymbol{\alpha}$ and using Bayes' theorem (3) $p(\boldsymbol{\alpha} \mid \boldsymbol{d}, \mathbf{X}, I)$ becomes proportional to the likelihood $p(\boldsymbol{d} \mid \boldsymbol{\alpha}, \mathbf{X}, \boldsymbol{s}, I)$. Assuming that the $r_{i}$ are samples from a Gaussian distribution with zero mean and variance $s_{i}^{2}$, the likelihood becomes

$$
p(\boldsymbol{d} \mid \boldsymbol{\alpha}, \mathbf{X}, \boldsymbol{s}, I)=\frac{\sqrt{\operatorname{det} \mathbf{C}}}{(2 \pi)^{N / 2}} \exp \left[-\frac{(\boldsymbol{d}-\mathbf{X} \boldsymbol{\alpha})^{T} \mathbf{C}(\boldsymbol{d}-\mathbf{X} \boldsymbol{\alpha})}{2}\right]
$$

where $\mathbf{C}$ is a diagonal matrix with elements $C_{i i}=1 / s_{i}^{2}$. Finally we need to specify $p(\boldsymbol{\alpha} \mid D, \boldsymbol{d}, \boldsymbol{\xi}, \mathbf{X}, I) \propto p(D, \boldsymbol{d} \mid \boldsymbol{\alpha}, \boldsymbol{\xi}, \mathbf{X}, \sigma, \boldsymbol{s}, I)$. Since the measurements $\boldsymbol{d}$ and $D$ are independent one gets for the latter

$$
p(D, \boldsymbol{d} \mid \boldsymbol{\alpha}, \boldsymbol{\xi}, \mathbf{X}, \sigma, \boldsymbol{s}, I)=p(D \mid \boldsymbol{\alpha}, \boldsymbol{\xi}, \sigma, I) \cdot p(\boldsymbol{d} \mid \boldsymbol{\alpha}, \mathbf{X}, \boldsymbol{s}, I) .
$$

Assuming again that $\rho$ of Eq. (6) is a sample from a Gaussian distribution with zero mean and variance $\sigma^{2}$ the likelihood for the new datum $D$ becomes

$$
p(D \mid \boldsymbol{\alpha}, \boldsymbol{\xi}, \sigma, I)=\frac{1}{\sqrt{2 \pi \sigma^{2}}} \exp \left[-\frac{1}{2 \sigma^{2}}\left(D-\boldsymbol{\xi}^{T} \boldsymbol{\alpha}\right)^{2}\right] .
$$

With the above settings the integrations in Eqn. (7) and (8) can be done analytically. After some algebra the Expected Utility results in

$$
\begin{aligned}
E U(\boldsymbol{\xi}) & =\frac{1}{2}\left[\ln (1+G)-\frac{G}{(1+G)^{2}}\right], \\
G & =\frac{\boldsymbol{\xi}^{T}\left(\mathbf{X}^{T} \mathbf{C X}\right)^{-1} \boldsymbol{\xi}}{\sigma^{2}} .
\end{aligned}
$$

The result does not depend on the data values of the existing data $\boldsymbol{d}$, since the model function Eq. (5) is linear in $\boldsymbol{\alpha}$. It depends, however, on the uncertainty of $\boldsymbol{d}$ as well as the uncertainty of $\mathbf{X}$ which are condensed in the matrix C. Moreover, the uncertainty $\sigma$ of the future datum and the respective experimental configuration $\boldsymbol{\xi}$ enter the final result.

An approximation to (14) has been obtained earlier by [MacKay(1991)]. The difference between this and our results is that $E U(\boldsymbol{\xi})$ vanishes quadratically as a function of $G$ in (14), while MacKay obtains a linear dependence due to the missing second term in the brackets in (14). 


\section{Results}

The principle of Data Adaptive Exploration shall now be implemented for a subgroup from the ISCDB [Yamada et al.(2005)]. This subset comprises 153 data from the W7-AS stellarator taken at a rotational transform of $t=1 / 3$ and is the only subset for which not only data but also associated uncertainties for every parameter $a, P, n, B$ and $W$ are available.

\subsection{Data Adaptive Exploration}

As mentioned before, the parameters of interest are now the scaling parameters $\boldsymbol{\alpha}$ of Eq. (1). The design parameters, however, are the quantities effective minor radius $a$, toroidal magnetic field $B$, the heating power $P$ and the line averaged density $n$.

According to Eq. (2), the scaling law model reads:

$$
\ln W=\alpha_{c}+\alpha_{a} \cdot \ln a+\alpha_{P} \cdot \ln P+\alpha_{n} \cdot \ln n+\alpha_{B} \cdot \ln B
$$

The design parameters are now given by $\boldsymbol{x}_{i}=\{1, \ln a, \ln P, \ln n, \ln B\}^{T}$. The existing data $\boldsymbol{d}$ is a set of logarithmised values of the energy content $\left\{\ln W_{i}\right\}$.

The uncertainty $s_{i}$ is calculated from simple error propagation law according to equation (15) with

$$
\begin{aligned}
s_{i}^{2}= & \left(s_{\ln W}^{i}\right)^{2}+\alpha_{0, a}^{2}\left(s_{\ln a}^{i}\right)^{2}+\alpha_{0, P}^{2}\left(s_{\ln P}^{i}\right)^{2} \\
& +\alpha_{0, n}^{2}\left(s_{\ln n}^{i}\right)^{2}+\alpha_{0, B}^{2}\left(s_{\ln B}^{i}\right)^{2} .
\end{aligned}
$$

The single uncertainties $s_{\ln W}^{i}, s_{\ln a}^{i}, s_{\ln P}^{i}, s_{\ln n}^{i}$ and $s_{\ln B}^{i}$ are the uncertainties of the logarithmised coordinates in the parameter space and can be calculated from the values given in the database accounting for the logarithmic transformation. Since a priori the scaling parameters $\boldsymbol{\alpha}_{0}$ are unknown in 16, values from the theoretical Lackner-Gottardi scaling [Lackner and Gottardi(1990)] are used as first estimates. Given these definitions, the expression for the Expected Utility (14) can now be evaluated.

In the following, a distinction is made between the measured value of $W, W^{e x p}$, and the theoretical value, calculated by the power scaling law, $W^{\text {theo }}$. The uncertainty of $W^{\text {exp }}$ is given by the measurement uncertainty of the diamagnetic energy $W$ and can be found in the database. For $W^{\text {theo }}$ the uncertainty is computed by error propagation according to Eq. (16). 
The scaling law for the 153 data sets from W7-AS is calculated by regression with

$$
\begin{aligned}
\ln W^{\text {theo }}= & (-0.831 \pm 0.082)+(2.264 \pm 0.093) \ln a \\
& +(0.452 \pm 0.035) \ln P+(0.440 \pm 0.022) \ln n \\
& +(0.618 \pm 0.049) \ln B
\end{aligned}
$$

These values for $\alpha_{i}$ differ from the findings of ISS04 since a sub-set of the data was taken. Using these parameters, $\ln W^{\text {theo }}$ can be calculated and compared with the experimental value $\ln W^{e x p}$.

The DAE allows now to determine the utility of individual measurements from a given data set. For this purpose, a selected measurement is deleted from the data set, and its utility is calculated with respect to the remaining $(q-1)$ data points by equation (14). Here, $\{\boldsymbol{\xi}, \sigma\}$ is the experimental configuration for the extracted datum. The remaining data sets are described by $\{\widehat{\mathbf{X}}, \widehat{\boldsymbol{s}}\}$. Note, that the EU calculated for different individual measurements is not comparable quantitatively, because the reference data set $\{\widehat{\mathbf{X}}, \widehat{\boldsymbol{s}}\}$ is different in every case. Fig. 1 shows the $\ln W^{\text {theo }}$ plot for all 153 data points. The expected utility of every single datum is expressed in the colour code. An average tendency of higher expected utilities at high $\ln W$ is clearly visible.

Since Eq. (14) incorporates all reference data the Expected Utility is a context sensitive quantity, it depends on what has been measured beforehand. Therefore, it is instructive to analyse the reason for the different EU values of the data points in Fig. 1.

W7-AS was operated at two values for the magnetic field, at $B \approx 2.54 \mathrm{~T}$ and at $B \approx 1.24 \mathrm{~T}$. While the maximum $\mathrm{EU}$ value is given for the smaller field, high values of the EU can be found for both $B$ values. The scatter of $\mathrm{EU}$ vs. $B$ allows one to conclude that there is no obvious $B$ dependence for the data set under investigation. A similar conclusion can be drawn for the dependence on the minor radius $a$ for which data cluster at values of $a \approx 0.175 \mathrm{~m}$ and $a \approx 0.12 \mathrm{~m}$.

Considerable variation in the parameter space shows up for the lineaveraged density $n$ and the heating power $P$. To show possible interdependencies, the EU is displayed as a function of both parameters in Fig. 2. High utilities are found in regions of high heating power and high densities. In these regions of the $P-n$ plane only a few data points are available. The point which is farthest away from the origin shows the highest utility. It appears that regions of high sampling density give small information gains. 


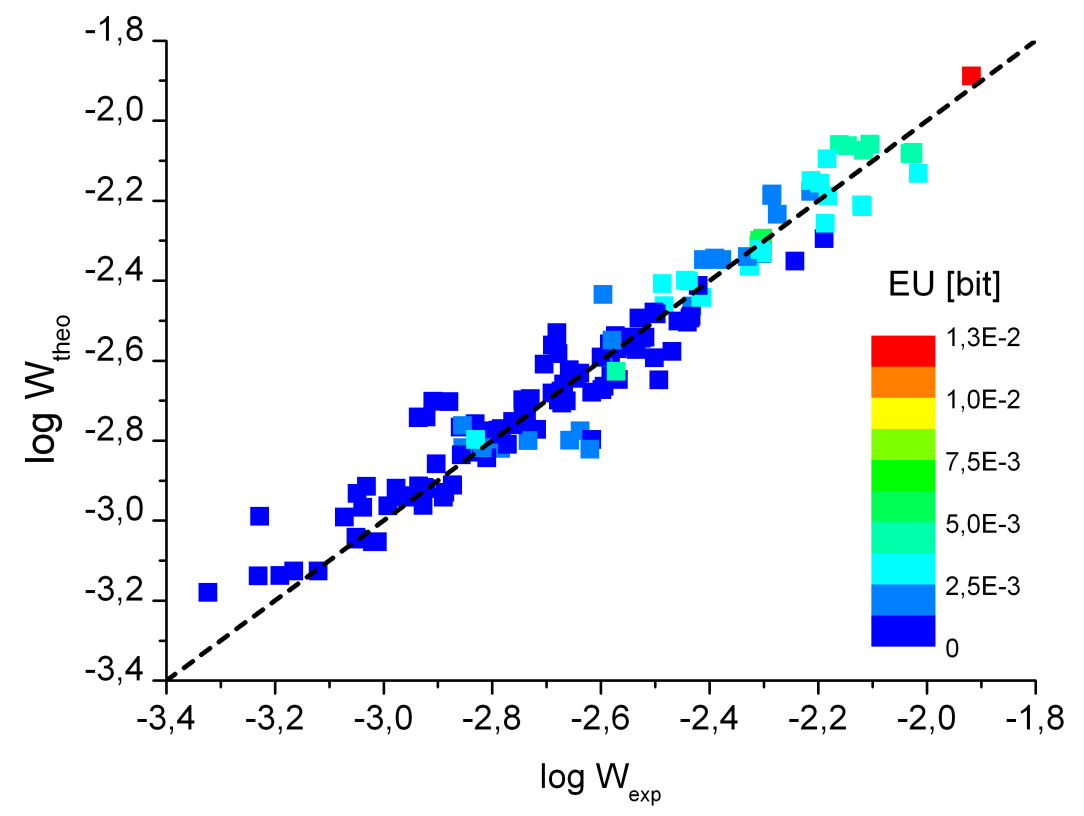

Figure 1: Comparison of $W^{e x p}$ and $W^{\text {theo }}$ for 153 data sets from W7-AS. The colour code expresses the utility of the respective data point. For comparison, the function $\log W^{\text {theo }}=\log W^{e x p}$ is also displayed (black line). 


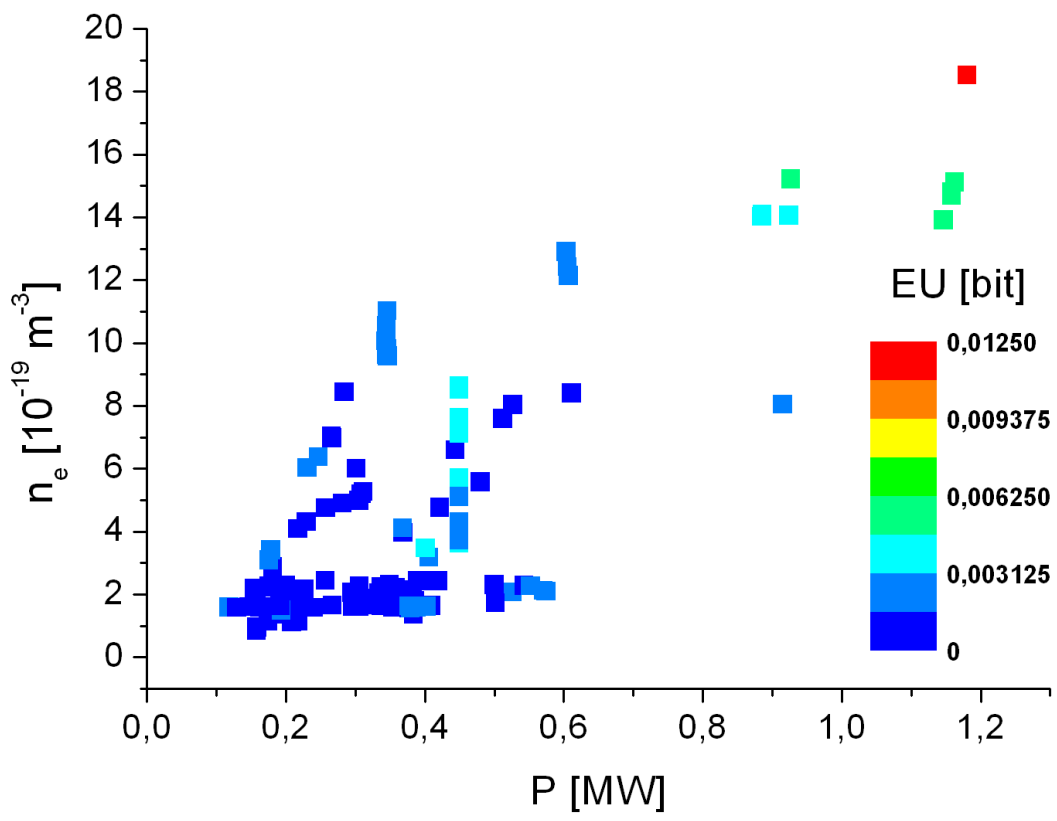

Figure 2: Power - density dependence of the utility, the EU is encoded in the colour scheme. 
As expected by common sense, a new measurement should lie in a parameter range not yet covered by previous experiments. This conclusion can be proven quantitatively by calculating the EU of a candidate new data point.

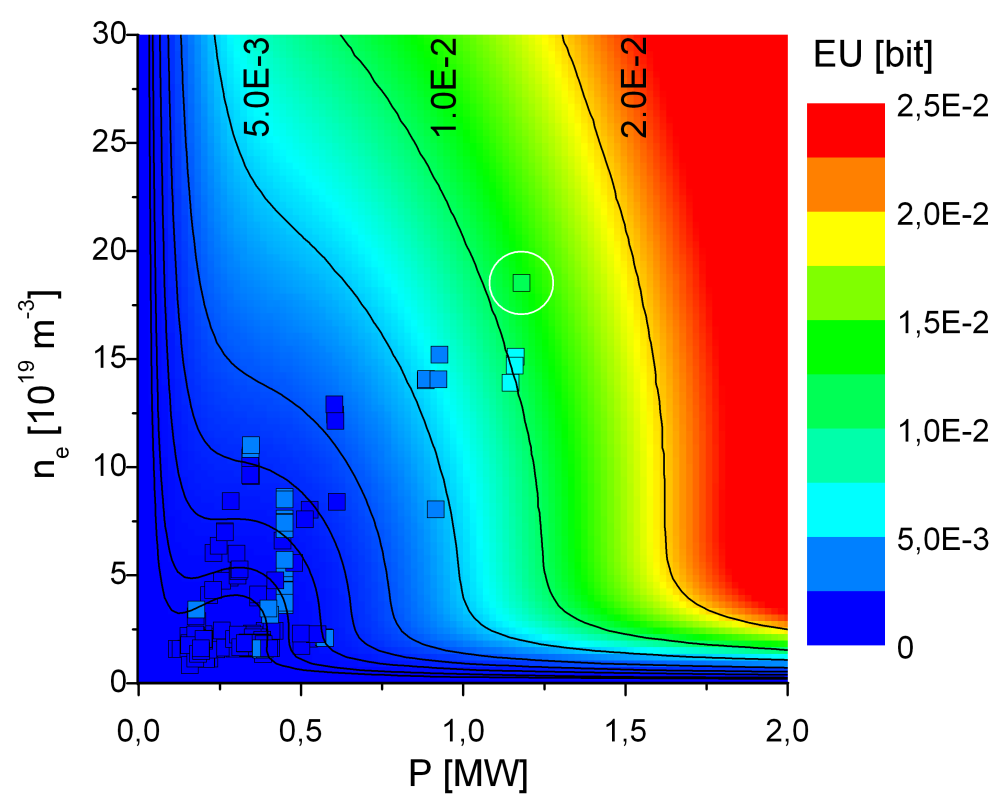

Figure 3: EU as a function of density $n$ and heating power $P$ for the W7-AS data set without the datum with the highest utility (encircled).

\subsection{Data Adaptive Planning}

For the planning of a future experiment the expected utility of the new data point is calculated. For the analysed W7-AS data set, the EU depends for the scaling law Eq. 2 in leading order on the heating power and the line averaged density. Therefore, the EU is determined in the $P-n$ plane.

The principle of planning is illustrated in Fig. 3. Here, the datum with the highest utility (see white circle in Fig. 3) was eliminated from the data set and the EU was determined for all $n$ and $P$ values yielding the two dimensional plot of EU vs $n$ and $P$. The EU of the erased datum was plotted for reference. This plot indicates parameter combinations for which 


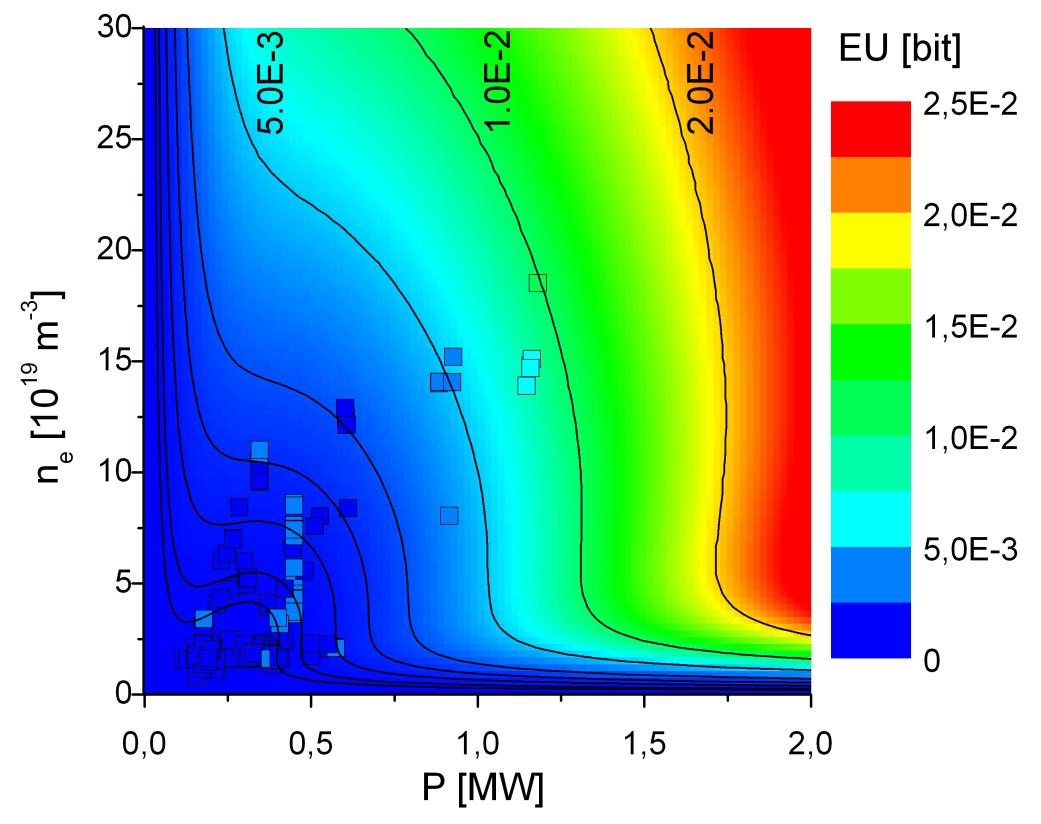

Figure 4: The same plot as Fig. 3, but now for a proposed 154th data point, given the 153 data sets from W7-AS. These sets are also shown for comparison. 
the information gain is expected to increase most. The EU for this new data point is lowest for small densities and heating powers. For intermediate densities $\left(2 \times 10^{19} \mathrm{~m}^{-3}<n_{e}<2 \times 10^{20} \mathrm{~m}^{-3}\right)$ the EU depends mostly on the heating power. Since there is no distinct maximum in Fig. 3, the planning strategy is to extend the covered parameter space into regions with highest $\mathrm{EU}$ values and the result indicates that a variation of density is much less informative than an increase of heating power.

In order to show the impact of new data on the planning procedure, the EU is now calculated for all data in Fig. 3. The EU was determined again in the $P-n$ plane, see Fig. 4 . For the proposed new data point, the parameter settings for the uncertainty, minor radius and magnetic field have been deliberately taken from the data point with the highest EU from the given data set.

The result confirms the conclusions from the previous example: The regions with a high EU are restricted to areas where no previous measurement exists. By comparison with Fig. 3 one also finds, that the area of smaller EU increases slightly around the data point with the highest EU. This shows the impact of this measurement on the EU distribution: Because this data point is now taken into account, measurements with similar $P$ and $n$ settings become less informative.

\subsection{Influence of the error statistics}

The influence of the measurement uncertainty on experimental planning shall be discussed next. In figure 5, the Expected Utility is given with respect to $W_{\text {theo }}$ and $W_{\text {exp }}$, whereas the uncertainty of $W_{\text {theo }}$, calculated by equation (16), is displayed as the size of the circles. As a tendency one finds that larger EUs correspond generally to smaller uncertainties (e.g. in case of the datum with the highest EU).

For further analysis of the influence of the measurement uncertainty on the EU, the change in the EU if no measurement uncertainty is specified. This assumption has been used frequently in the analysis of energy confinement in fusion devices. To eliminate the influence of the measurement uncertainty, the diagonal elements of the uncertainty matrix $\mathbf{C}$ and the uncertainty of the new datum are set equal to one. The results of these assumptions are shown in figure 6 which should be compared to figure 3. The utility for the given data changes dramatically if the influence of the measurement uncertainty is neglected. The shape of the EU distribution shows significant differences in 


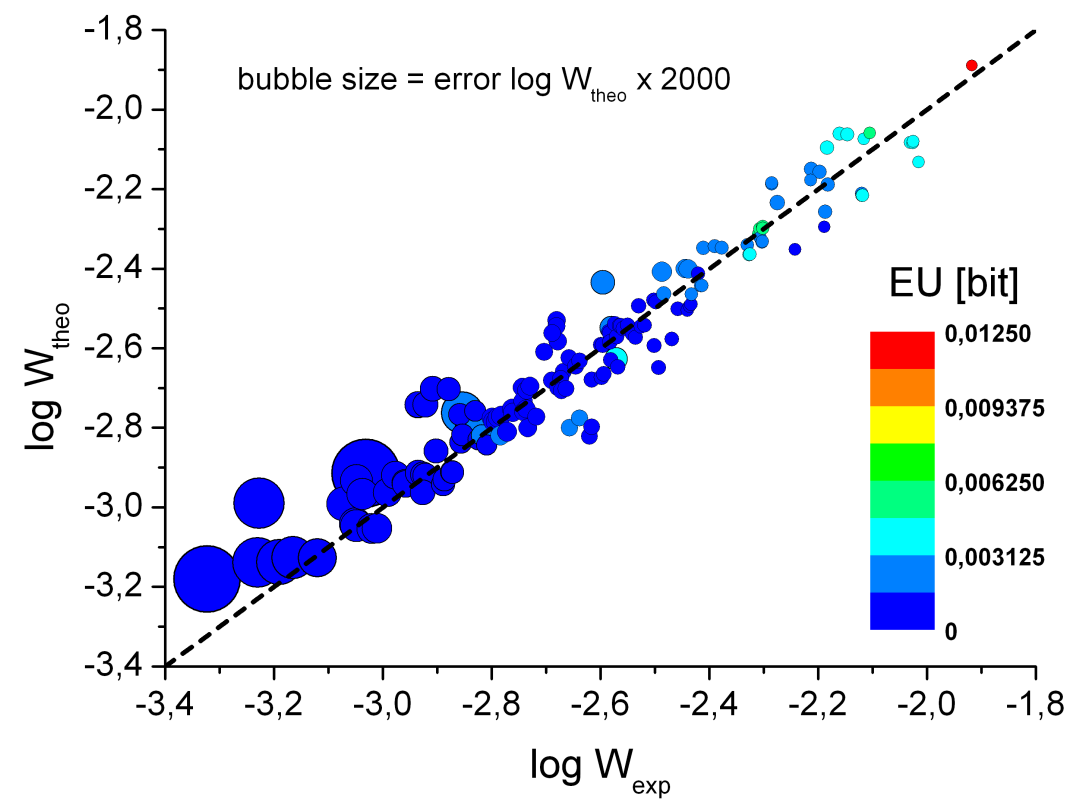

Figure 5: Comparison of $W^{e x p}$ and $W^{\text {theo }}$ for 153 data sets from W7-AS, the EU is colour coded. The symbol size is given by the uncertainty of $\ln W^{\text {theo }}$, scaled by a factor of 2000 .

the range of small values for $n$ and $P$ : Whereas in the case of correct treatment of the uncertainties the $\mathrm{EU}$ is low for these regions in parameter space, the values of the EU become large if uncertainties are neglected. The reason for this behavior is that an absolute constant uncertainty in primary space (cf. Eq. 1) transforms into a relative uncertainty in logarithmic space with the consequence that measurements at small $n$ and $P$ become as important as the measurements at larger values. This offends common sense.

One also finds that the EU of the given 153 data points changes in comparison to figure 3: E.g., the datum with the maximum EU from this figure shows a strong decrease in its utility if the uncertainty is neglected. The effect can be explained as follows: The measurement uncertainty of this data set is relatively small as shown in Fig. 5. Therefore, the EU value indicates the datum to be more valuable compared to other data points. This conclusion cannot be drawn if the measurement uncertainties are slighted all together. 


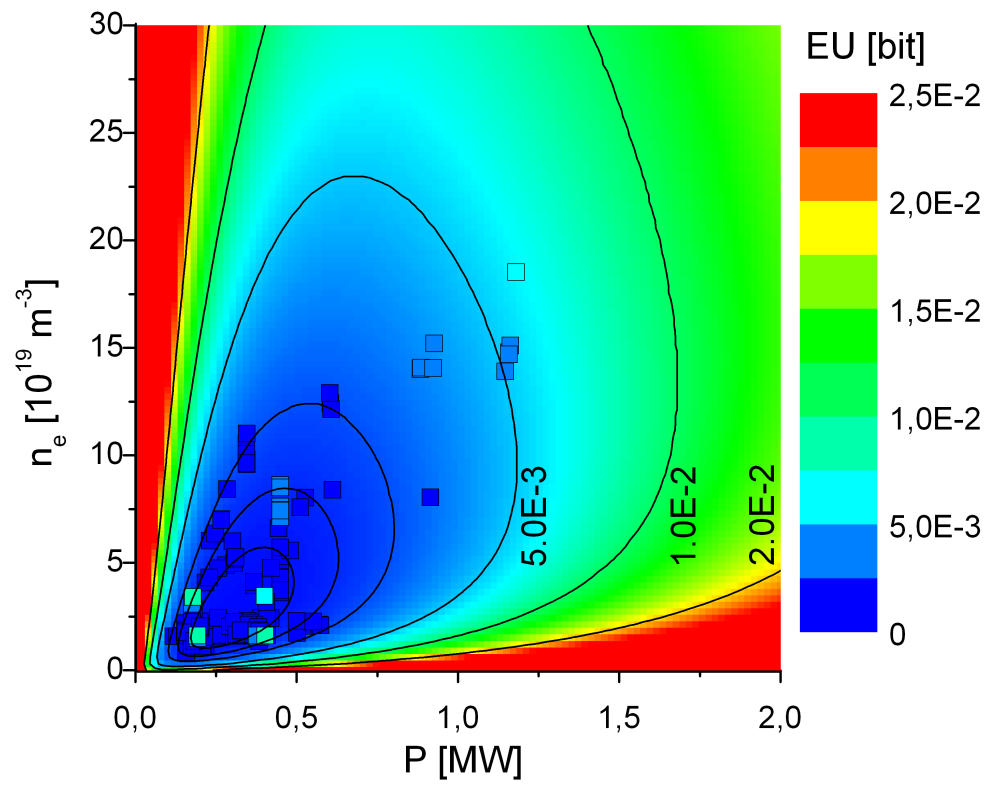

Figure 6: The influence of the measurement uncertainty on the EU for scaling laws: EU distribution for the encircled datum in figure 3, but now the uncertainty was taken equal 1 for all data points (no effect of considering the uncertainty).

One can conclude here that the influence of the measurement uncertainty is significant, data points with large uncertainties are not beneficial. Therefore, the measurement uncertainty has to be taken into account and its value has to be estimated with due care.

\section{Conclusions}

In this paper it was shown that the Bayesian approach can be used for the planning of a future measurement, taking into account a data base with already measured data. Given a multi variate linear physical problem, the Expected Utility of a new datum depends on the experimental configuration of the existing data sets and of the new datum. Also, the EU is significantly influenced by the measurement uncertainty of the new datum. It has often 
been claimed in Bayesian data analysis that the uncertainty of the data are as important as the data themselves. Here we have the extreme situation that the results of an analysis do not depend anymore on $\boldsymbol{d}, D$ but only on $\boldsymbol{s}, \sigma$. This result which goes much beyond the specific problem treated here but is of general interest.

It can easily be seen from Eq. (14) that the EU decreases with an increasing measurement uncertainty $\sigma$. Consequently, for a future experiment the measurement uncertainty should be kept as small as possible.

Concerning the dependency on the experimental configuration one can conclude that measurements in regions of the configuration space with a sparse number of given data points show the highest Expected Utility, in other words, one should choose experimental configurations which have not been applied before to gain maximum information. This agrees with common sense.

For the planning of the next measurement, an experimental configuration with high EU should be chosen. Not every configuration may be realisable, technical and/or physical constraints may limit the configuration space. Furthermore, one can also implement a cost function which expresses the necessary effort for the realisation of a certain parameter combination. The optimal experimental configuration is then given by the best relation of cost and expected information gain.

For the problem presented here, the $\mathrm{EU}$ is independent of the data values from the data base. This allows one to design experimental campaigns with more than one new measurement: If the experimental conditions of the next measurement were estimated by DAP this configuration can be added to the existing data set. Then, the next experiment can be planned using the DAP approach, and so on. The only assumption to be made is about the measurement uncertainty of future experiments, which may be estimated from experience with previous measurements. With this method, more than one future experiment can be planned. Therefore, the approach of DAP can also be used for the planning of full experimental campaigns.

\section{Acknowledgments}

The authors appreciate helpful discussions with R. Fischer. This work was conducted within Sonderforschungsbereich TR24 (Project B8A). 


\section{References}

[ITER team(1999)] ITER team, Nucl. Fusion 39, 2137 (1999).

[Lindley(1956)] D. Lindley, The Annals of Math. Stat. 27, 986 (1956).

[Loredo(2003)] T. Loredo, in Bayesian Inference and Maximum Entropy Methods in Science and Engineering, edited by G. Erickson and Y. Zhai (AIP Conference Proceedings, Melville, N.Y., 2003), pp. 330-346.

[Dreier et al.(2006)] H. Dreier et al., Fusion Sci. Technol. 50, 262 (2006).

[Yamada et al.(2005)] H. Yamada et al., Nucl. Fusion 45, 1684 (2005).

[Dose et al.(1996)] V. Dose et al., Nucl. Fusion 36, 735 (1996).

[Jaynes(2005)] E. T. Jaynes, The Logic of Science (Oxford University Press, Oxford, 2005).

[Kapur and Kesavan(1992)] J. N. Kapur and H. K. Kesavan, Entropy Optimization Principles with application (Academic Press Inc., Boston, MA, 1992).

[MacKay(1991)] D. MacKay, Neural Computation 4, 589 (1991).

[Lackner and Gottardi(1990)] K. Lackner and E. Gottardi, Nucl. Fusion 30, 76 (1990). 